\title{
Scintillation Measurement on Ku-band Satellite Path in Tropical Climate
}

\author{
Hassan Dao, Islam Md. Rafiqul, Jalel Chebil, and Khalid Al-Khateeb \\ Department of Electrical and Computer Engineering \\ International Islamic University Malaysia \\ Jalan Gombak, 53100 Kuala Lumpur, Malaysia \\ sun.spu@gmail.com,rafiq@iium.edu.my
}

\begin{abstract}
Scintillation data was collected in Kuala Lumpur, Malaysia for one year measurement. The data were obtained from MEASAT3 satellite operating at Ku-band with elevation angle of $77.4^{\circ}$. In this paper, scintillation statistics are analysed in dry (non-rain) condition. The scintillation distributions are represented scintillation intensity and amplitude by monthly, seasonal, worst-month and annual distributions. Probability density function (PDF) of scintillation intensity correspondingly agrees to Generalize Extreme Value (GEV) fit. In addition, model validation to the measured data is also provided.
\end{abstract}

Keywords-scintillation amplitude and intensity; distribution statistics; tropical climate;

\section{INTRODUCTION}

Scintillation is fast fluctuation in amplitude and phase of the millimetre-wave satellite signal. Scintillation is due to turbulence in atmosphere. It can impair satellite transmission in frequency bands above $3 \mathrm{GHz}$ [1]. Therefore, tropospheric scintillation is a crucial factor that can degrade radiowaves operating frequencies at Ku-band and higher bands. The fluctuations due to scintillation increase with frequency, depend upon the slant path length, especially low elevation angle, decrease with the antenna beamwidth and significantly in low fade-margin system [2, 3]. Furthermore, scintillation is also dependent on meteorological parameters such as temperature, humidity and refractive index variations of propagation media.

Statistical knowledge of scintillation must be therefore comprehensible for planning and designing in satellite system. In this paper, scintillation statistics are presented for different time scales of monthly, season, worst-month and annual average distribution statistics by successfully removing rain events.

\section{EXPERIMENTAL SETUP AND DATA PROCESSING}

The MEASAT3 $\left(91.5^{\circ}\right)$ signal is monitored in Ku-band centered around the frequency $10.982 \mathrm{GHz}$ for 12 consecutive months ranging from $1^{\text {st }}$ September 2011 till $31^{\text {st }}$ August 2012 with a data availability of $97.8 \%$. The measurement system is located at the faculty of Engineering, International Islamic university Malaysia (IIUM), Kuala Lumpur, Malaysia. The signal sampling frequency is $10 \mathrm{~Hz}$ and subsequently processed to $1 \mathrm{~Hz}$ sampling frequency. Raining events were manually removed based on $0.2 \mathrm{~mm}$ tipping bucket rain gauge which is time synchronized measurement. Details of the experimental setup are available in $[4,5]$.

Power spectral density (PSD) is an efficient technique to determine a filtering process. The cutoff frequency, $f_{B}$ of 78 $\mathrm{mHz}$ is determined to separate rain attenuation and tropospheric scintillation data [5]. The LPF with $f_{B} 78 \mathrm{mHz}$ is utilised to extract rain attenuation from the attenuation time series while a Butterworth band pass filter (BPF) with frequency bandwidth of $0.078-0.5 \mathrm{~Hz}$ is utilised to obtain scintillation amplitude and avoid system noise floor [6]. All rain events recorded in rain gauge were used to separate rain attenuation data from this analysis.

\section{SCINTILLATION INTENSITY AND VARIANCE DisTRIBUTION STATISTICS}

Scintillation intensity $\sigma_{\chi}(\mathrm{dB})$ is usually described by the short-term of scintillation amplitude $\chi$. The parameter is estimated by calculating standard deviation of scintillation amplitude $\chi$ over one-minute interval from the attenuation of time series. Monthly mean value of measured scintillation intensity $\sigma_{\chi}$ is showed in Fig. 1.

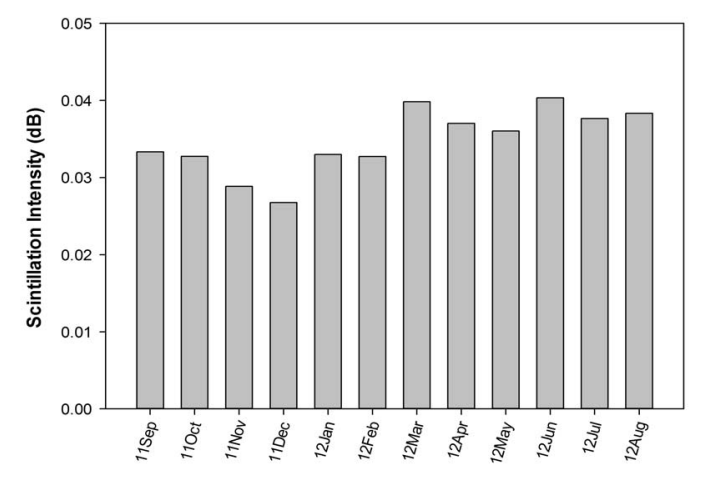

Fig. 1. Monthly mean values of measured scintillation intensity. 


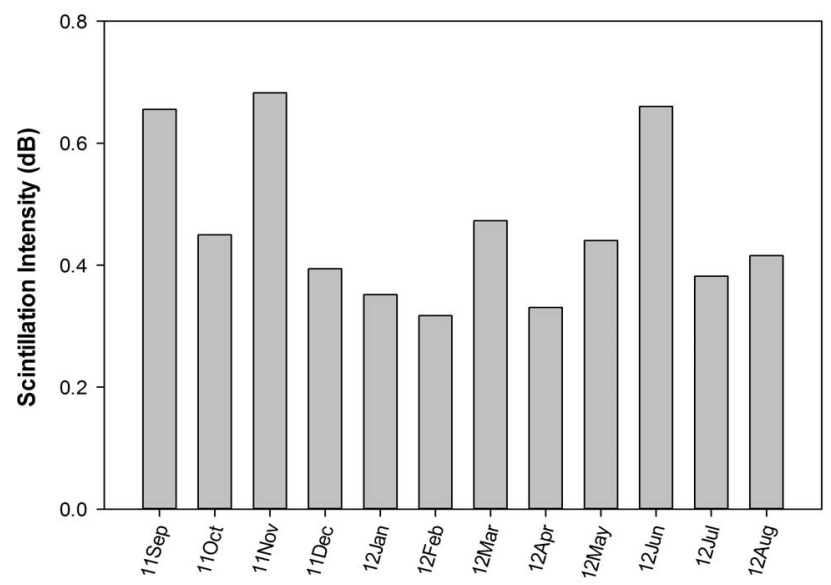

Fig. 2. Monthly maximum occurrence of measured scintillation intensity

Malaysia is located in a tropical region that has heavy rain throughout the year. Climate distributions can be characterised by monsoon behaviours. The Northeast monsoon or called Wet season is during October and March whereas Southwest monsoon is through April and September called Dry season [7]. The average scintillation intensity $\sigma_{\chi}$ has generally stronger in dry season than wet season. The scintillation intensity $\sigma_{\chi}$ reaches maximum of $0.68 \mathrm{~dB}$ in November and following by $0.66 \mathrm{~dB}$ in June. The monthly maximum of measured scintillation intensity is depicted in Fig. 2.

Scintillation variance, $\sigma_{\chi}^{2}\left(\mathrm{~dB}^{2}\right)$ could be better to represent distribution statistic characteristic of scintillation intensity, $\sigma_{\chi}$. Scintillation variance, $\sigma_{\chi}^{2}\left(\mathrm{~dB}^{2}\right)$ is computed by the square of the scintillation standard deviation over one-minute interval. The monthly average cumulative distribution of the variance $\left(\mathrm{dB}^{2}\right)$ is estimated from measured data and presented in Fig. 3. The strongest cumulative statistics of $\sigma_{\chi}^{2}$ is mainly occurred in June for the strength below $0.26 \mathrm{~dB}^{2}$ and in November for $\sigma_{\chi}^{2}$ above $0.26 \mathrm{~dB}^{2}$.

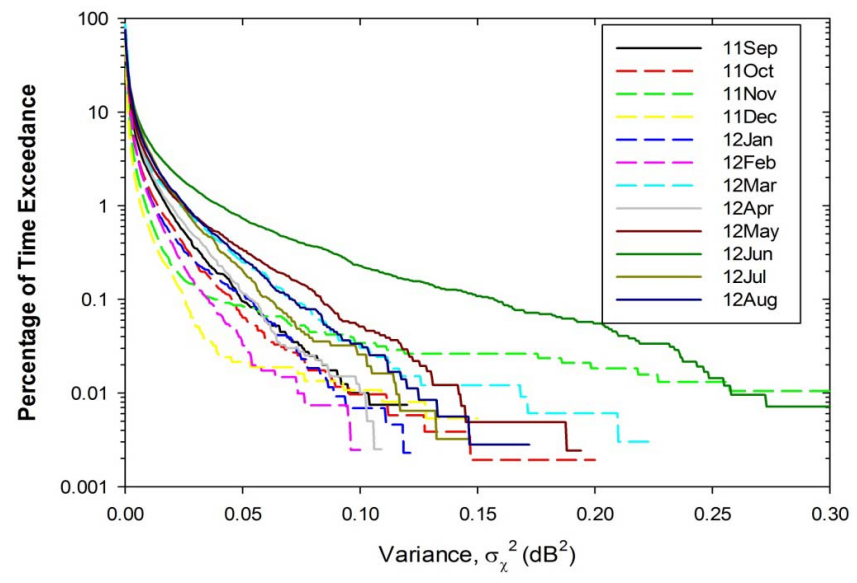

Fig. 3. Monthly cumulative distribution of scintillation variance $\left(\mathrm{dB}^{2}\right)$

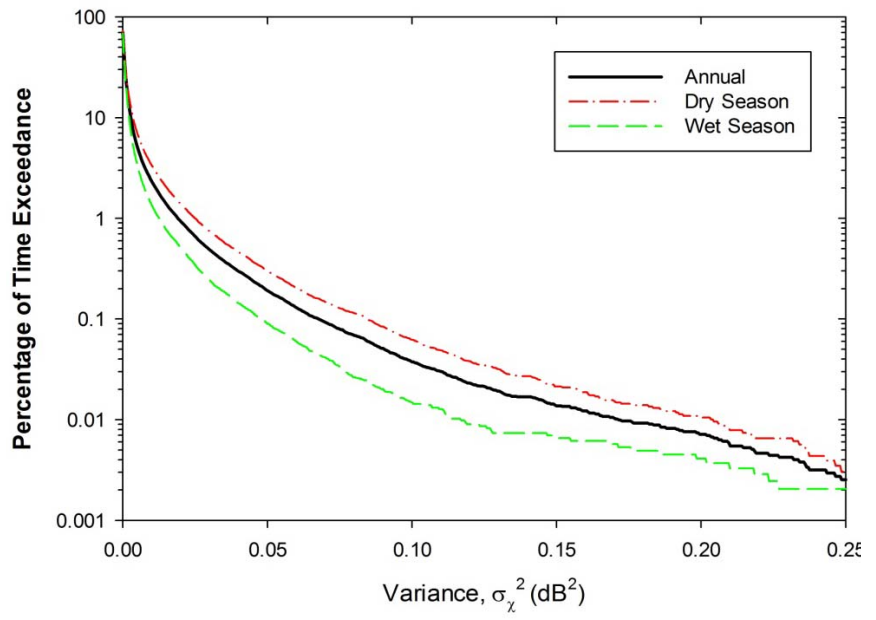

Fig. 4. Annual and seasonal cumulative distributions of scintillation variance $\left(\mathrm{dB}^{2}\right)$ measured from September 2011 to August 2012.

Fig. 4. shows the cumulative distribution of seasonal and annual average scintillation variance $\sigma_{\chi}^{2}$. Scintillation strength is stronger during dry season compared to wet season. For instance, $\sigma_{\chi}^{2}$ value is $0.11 \mathrm{~dB}^{2}$ in wet season while $0.19 \mathrm{~dB}^{2}$ in dry season for percentage of time at $0.01 \%$. From the result shown, the scintillation strength is correspondingly higher humidity and $N_{\text {wet }}$ in dry season.

The probability density function (PDF) of dry scintillation intensity, namely lognormal, gamma and Generalized extreme value (GEV) fits is compared to measured one-year scintillation by Statistical fitting tool in Matlab. The results are presented in Fig. 5. The GEV could be determined by (1-2) [2, 8].

For $\mathrm{k} \neq 0$

$p\left(\sigma_{\chi}\right)=\frac{1}{\gamma}\left[1+k\left(\frac{\sigma_{\chi}-\mu}{\gamma}\right)\right]^{-1-\frac{1}{k}} \cdot \exp \left(-\left(1+k\left(\frac{\sigma_{\chi}-\mu}{\gamma}\right)\right)\right)^{-1 / k}$

and for $\mathrm{k}=0$

$p\left(\sigma_{\chi}\right)=\frac{1}{\gamma} \exp \left[-\exp \left(-\frac{\sigma_{\chi}-\mu}{\gamma}\right)-\left(\frac{\sigma_{\chi}-\mu}{\gamma}\right)\right]$

Where: $k$ is the shape parameter $(0.336)$,

$\gamma$ is scale parameter $(0.0097)$,

and $\mu$ is location parameter $(0.0242)$. 


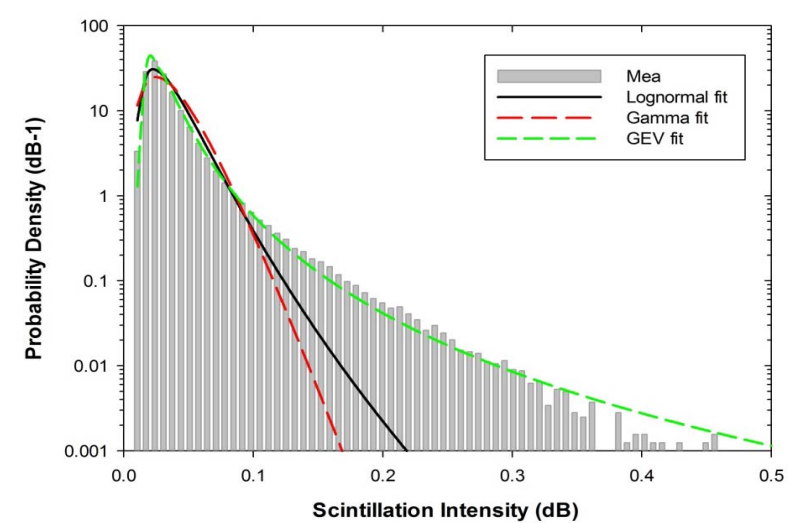

Fig. 5. Measured probability density of scintillation intensity with lognormal, gamma and generalized extreme value (GEV) fits.

The measured PDF discernibly fitted well with lognormal and gamma best fits for $\sigma_{\chi}$ below $0.1 \mathrm{~dB}$ and evidently underestimated for $\sigma_{\chi}$ above $0.1 \mathrm{~dB}$. The GEV found the best performance to fit empirical data.

Table I. RMS of percentage of fractional error for PDF of scintillation intensity

\begin{tabular}{|l|r|r|r|r|}
\hline & Measured & Lognormal & GEV & Gamma \\
\hline RMS & 5.98 & 5.50 & 6.05 & 5.06 \\
\hline Error (\%) & & 8.03 & -1.16 & 15.40 \\
\hline
\end{tabular}

Root mean square (RMS) of percentage fractional error, $\varepsilon$ between measurement and prediction fit can be calculated as follow [9].

$$
\varepsilon=\frac{\sigma_{\chi(\text { mea })}-\sigma_{\chi(\text { pre })}}{\sigma_{\chi(\text { mea })}} \times 100 \%
$$

Table I presents RMS of percentage fractional error, $\varepsilon$ for dry scintillation intensity calculated by (3). The result shows that lognormal and gamma fit underestimated the measured data with $8.03 \%$ and $15.4 \%$, respectively whereas GEV overestimated only $1.16 \%$.

\section{ANALYSIS OF SCINTILLATION AMPLITUDE}

Long-term scintillation amplitude is processed only during clear-sky events. Cumulative distribution functions (CDF) of monthly, seasonal, worst-month and annual aspects are presented in this section with scintillation enhancement amplitude (positive log-amplitude) and fade amplitude (negative log-amplitude).

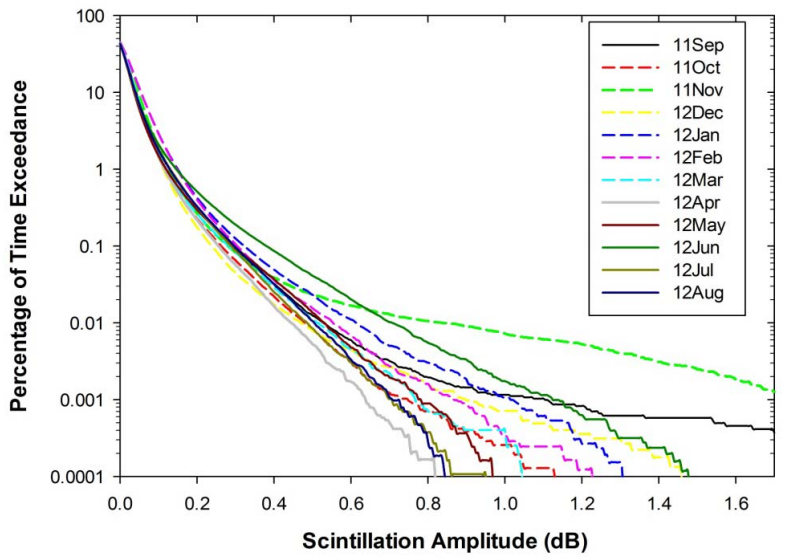

Fig. 6. Monthly cumulative distribution of scintillation fades from one-year measurement.

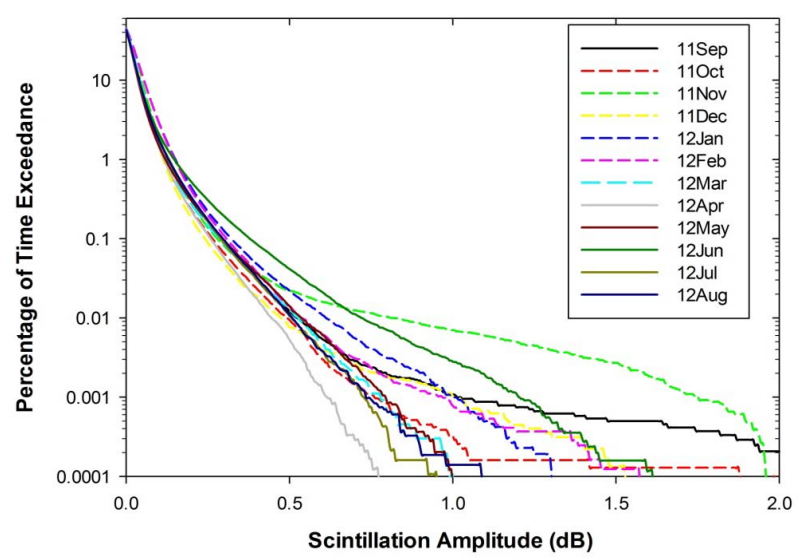

Fig. 7 Monthly cumulative distribution of scintillation enhancement from oneyear measurement.

Fig. 6 and 7 depict the monthly CDF of scintillation fade and enhancement, respectively for the twelve consecutive months period. The cumulative distribution of scintillation fade and enhancement are highest in June and November for the amplitudes below and above $0.65 \mathrm{~dB}$, respectively. The highest peak occurrences of measured scintillation fade and enhancement were in September for 3.5 and $3.02 \mathrm{~dB}$, respectively. It is obvious that at $0.01 \%$, scintillation fade and enhancement varied around 0.5 to $0.85 \mathrm{~dB}$ all the months. However, variation of scintillation fade and enhancement at $0.1 \mathrm{~dB}$ and above is lower percentage of time. Hence scintillation for high elevation angle links at Ku-band is significant for highly reliable system. 


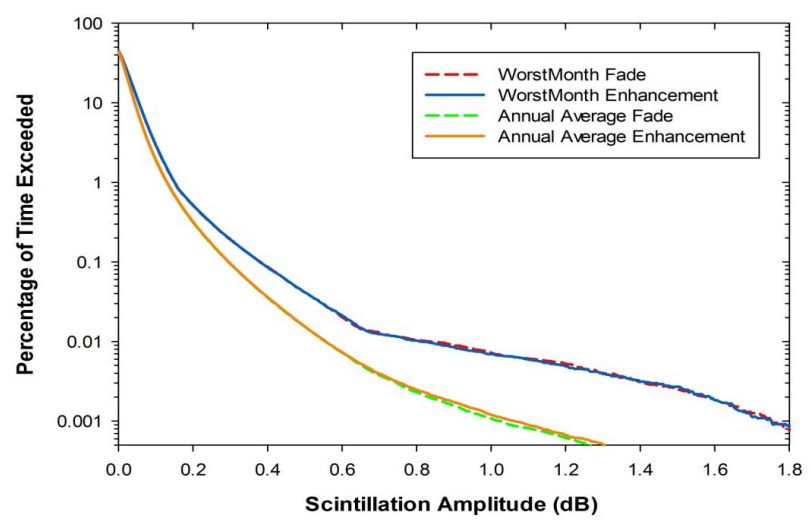

Fig. 8. Comparison of worst-month and annual cumulative distributions of scintillation amplitude.

Worst-month statistics for both scintillation fade and enhancement are estimated and compared with annual average statistics. The worst-month concept is conversion of the margin exceedance of twelve consecutive calendar months that is referred as the annual worst-month time fraction of excess [10]. Fig. 8 presents the scintillation amplitude distributions of worst-month and annual average statistics. Scintillation fade and enhancement had similar shapes. The annual enhancement is slightly higher than fade for above exceedance of $0.06 \%$. During this threshold is exceeded for the longest time that to consider the performance degrading mechanism. The result shown the worst-month is margin of exceedance in June and November for amplitude below and above $0.65 \mathrm{~dB}$ (equivalent $0.09 \%$ exceedance), respectively. For $0.01 \%$ of time exceedance, the annual average is about $0.6 \mathrm{~dB}$ for both fade and enhancement while worst-month reached up to $0.9 \mathrm{~dB}$.

\section{CONCLUSION}

Scintillation is dependent on meteorological parameters, especially temperature, humidity and wet-term refractivity. Scintillation amplitude and intensity distributions are measured, analysed and presented in details. In general, scintillation enhancement is found slightly higher than scintillation fade. Scintillation in dry season is evidently stronger than wet season for both clear air and rainy conditions. The worst-month of scintillation is exceeded margin in June and November for amplitude below and above $0.65 \mathrm{~dB}$, respectively.

Short term of scintillation amplitude called scintillation intensity (standard deviation) is estimated by taken 60 blocks (1minute) of data. Probability density function (PDF) of scintillation intensity agrees with generalized extreme value (GEV), and better than lognormal and gamma fits.

\section{ACKNOWLEDGMENT}

This work is supported by the Research Management Centre (RMC), project no. EDW B13-038-0923, International Islamic University Malaysia (IIUM).

\section{REFERENCES}

[1] J. L.J. Ippolito, Satellite Communications Systems Engineering: John Wiley and Sons, 2008.

[2] P. Garcia-del-Pino, J. M. Riera, and A. Benarroch, "Tropospheric Scintillation With Concurrent Rain Attenuation at $50 \mathrm{GHz}$ in Madrid," Antennas and Propagation, IEEE Transactions on, vol. 60, pp. 15781583, 2012.

[3] C. N. Kassianides and I. E. Otung, "Dynamic model of tropospheric scintillation on earth-space paths," Microwaves, Antennas and Propagation, IEE Proceedings -, vol. 150, pp. 97-104, 2003.

[4] H. Dao, M. R. Islam, and K. Al-Khateeb, "Modification of ITU-R Rain Fade Slope Prediction Model Based on Satellite Data Measured at High Elevation Angle," IIUM Engineering Journal (IIUMEJ), vol. 12, pp. 53-59, 2011.

[5] H. Dao, M. R. Islam, and K. Al-Khateeb, "Rain Fade Slope Model in Satellite Path Based on Data Measured in Heavy Rain Zone," Antennas and Wireless Propagation Letters, IEEE, vol. 12, pp. 50-53, 2013.

[6] E. Matricciani and C. Riva, "18.7 GHz tropospheric scintillation and simultaneous rain attenuation measured at Spino d'Adda and Darmstadt with Italsat," Radio Science, vol. 43, pp. 1-13, 2008.

[7] J. S. Mandeep, "Equatorial rainfall measurement on kuband satellite communication downlink," Progress In Electromagnetics Research, vol. 76, pp. 195-200, 2007.

[8] S. Kotz and S. Nadarajah, Extreme Value Distributions: Theory and Applications. London: Imperial College Press, 2000.

[9] P. Yu, P.A. Watson, O.T. Davies, S. Ventouras, and C. Wrench, "Review and comparison of tropospheric scintillation prediction models for satellite communications," International journal of satellite communications and networking, vol. 24, pp. 283-302, 2006.

[10] ITU-RP.581-2, "The Concept of Worst-Month," International Telecommunications Union, Geneva1990. 